\title{
Making the user more efficient: Design for sustainable behaviour
}

\author{
Dan Lockton ${ }^{\mathrm{a} 1}$, Professor David Harrison ${ }^{\mathrm{a}}$ and Professor Neville Stanton ${ }^{\mathrm{a}}$
}

${ }^{a}$ School of Engineering \& Design, Brunel University, Uxbridge, Middlesex, UK

\begin{abstract}
User behaviour is a significant determinant of a product's environmental impact; while engineering advances permit increased efficiency of product operation, the user's decisions and habits ultimately have a major effect on the energy or other resources used by the product. There is thus a need to change users' behaviour. A range of design techniques developed in diverse contexts suggest opportunities for engineers, designers and other stakeholders working in the field of sustainable innovation to affect users' behaviour at the point of interaction with the product or system, in effect 'making the user more efficient'. Approaches to changing users' behaviour from a number of fields are reviewed and discussed, including: strategic design of affordances and behaviour-shaping constraints to control or affect energyor other resource-using interactions; the use of different kinds of feedback and persuasive technology techniques to encourage or guide users to reduce their environmental impact; and context-based systems which use feedback to adjust their behaviour to run at optimum efficiency and reduce the opportunity for user-affected inefficiency. Example implementations in the sustainable engineering and ecodesign field are suggested and discussed.
\end{abstract}

Keywords: ecodesign; sustainability; managing use; managing consumption; behaviour change; sustainable innovation; persuasive technology

\section{Introduction}

For many consumer products, the use phase is the most significant in terms of environmental impact, primarily energy use. Technological responses to mitigate this impact form a substantial proportion of work in the sustainable engineering field: increased efficiency of operation and reduction of waste generated are important goals.

It may also be equally — and independently — worthwhile to reduce or otherwise alter the manner or period of products' use, which implies changing users' behaviour. Government policy responses, in the form of educational, economic and legal measures, often aim to address this issue, but design and engineering methods also have potential to assist in persuading or guiding users to operate products in a more sustainable manner, following the intentions of the designer to self-manage use and resource consumption. In effect, it is possible to 'make the user more efficient'. This

\footnotetext{
${ }^{1}$ Corresponding author. Email: Daniel.Lockton@brunel.ac.uk
}

NOTE: This is a preprint of an article whose final and definitive form will be published in the International Journal of Sustainable Engineering. 
article reviews some approaches to designing for sustainable behaviour from different disciplines, and their applicability to sustainable engineering and ecodesign.

\section{Approaches to design for sustainable behaviour: 'Design with Intent' thinking}

The idea of using features of a system - a physical product, built environment, computer network, or indeed any system with which a user interacts - to guide, shape or regulate the ways in which interaction occurs has been expressed in many ways in a number of different fields, and might be loosely described as 'Design with Intent' (DwI) thinking (Lockton, 2008). The term behaviour-steering design has also been used, starting from a sociological context (Jelsma 2000, 2006). A common factor is that this is strategic design intended to result in certain user behaviour: there is intent on the part of the designer.

While in a sustainable engineering and ecodesign context, reducing energy and resource use is the priority, it makes sense to review a wider scope of DwI examples and approaches where the intent can be commercially or socially motivated (or both). Many of the techniques employed in other contexts can be abstracted and applied to sustainable engineering problems.

\subsection{Affordances, constraints and mistake-proofing}

Many DwI approaches involve the planning and strategic manipulation of the affordances (including perceived affordances) and constraints of a system. That is, the actions or functions which are offered or presented to users (or which they perceive are available to them) and the constraints or limits on their behaviour provided by the system.

The theory of affordances was outlined by the ecological psychologist James Gibson (1979, p. 127-143) and developed by Donald Norman (1988, p. 9-11), focusing primarily on users' perceptions of the affordances available to them, and how to improve product and interface usability by understanding this aspect of design. A ubiquitous example of the power of perceived affordances is the use of handles or plates on doors to signal whether they should be pulled or pushed; the frequent passing frustrations of visitors to unfamiliar buildings on finding that their intuitions are incorrect, and a door whose handle appears to say "pull me" actually requires the handle to be pushed, demonstrate how deeply rooted and influential affordances can be in shaping our everyday behaviour.

The manner in which the affordances of a system are presented to users, and which possible actions are made more prominent - including aspects such as the choice of defaults (Kesan \& Shah 2006) - will have an impact on the choices users make: in the environmental field, a common example is the increasing adoption of 'economy' 30 or 40-degree wash cycles on domestic washing machines. If the options are available, and easy for users to select, it is more likely that they will be used. Where energy-using products (household appliances) have multiple possible energy

NOTE: This is a preprint of an article whose final and definitive form will be published in the International Journal of Sustainable Engineering.

(C) 2008 Taylor \& Francis; International Journal of Sustainable Engineering is available online at: http://journalsonline.tandf.co.uk/ 
use modes or settings (e.g. a refrigerator with adjustable temperature, or a washing machine with a number of different wash cycles), the system could default to the mode which uses the least energy, and thus require users to make an explicit choice to deviate from this. Depending on the complexity of the system, a context-based approach (see section 2.3 below) may be more appropriate, to reduce the likelihood of a too-low setting (e.g. a short 30 degree wash cycle applied to heavily stained clothes) giving poor results, leading to frustration. If there is no default mode, simply making the least energy mode more prominent or easy to select is an alternative that can be accomplished simply by redesigning the user interface (equally, making energyintensive modes more difficult to select may achieve the same result). Devices where unnecessary (excessive) energy and water use are very common, such as electric kettles, could require users to make a choice about the amount of water that needs to be heated before starting, as on the Eco Kettle (Product Creation Ltd., n.d.) or heat only one unit quantity at a time, as on the Tefal QuickCup (Tefal UK Ltd., n.d.)

Norman (1988) also describes various behaviour-shaping constraints, including forcing functions such as interlocks (where one condition must be satisfied before a function is enabled); this type of constraint is probably most familiar to many engineers through the use of poka-yoke (Japanese: 'mistake-proofing') in manufacturing industry — defensive design techniques originally developed by Shigeo Shingo in the context of the Toyota Production System, intended to ensure 'zero defects' in assembly processes (Shingo 1986; Nikkan Kogyo Shimbun, Ltd. \& Factory Magazine 1989). 'Control' poka-yokes, such as lock-ins, lock-outs and interlocks, are designed to prevent errors occurring, by making it impossible or difficult to proceed until the error is corrected - in a sustainable engineering context, these can allow 'inefficient' operating procedures to be prevented. For example, automatic lighting and water taps only operate when a user is present. 'Warning' poka-yokes, for example lights, buzzers, information displays and even reminders of various kinds alert users to the presence of an error, abnormal condition or extra step which must be performed. These 'warning' poka-yokes are perhaps better considered along with other methods of persuasion (see section 2.2 below). Other methods such as the use of go/no-go gauging, templates and jigs (Chase \& Stewart, 2007) may fall somewhere between 'warning' and 'control', alerting the user but not always (depending on context) preventing the operation continuing.

It is worth noting that even a very simple division of errors into 'mistakes' (where the intended action was a priori inappropriate or incorrect) and 'slips' (where the intention was correct but the action was performed incorrectly) (Norman \& Lewis 1986), introduces another dimension to the field, with mistakes often resulting from an incorrect or incomplete mental model of the system on the part of the user. This is relevant in sustainable engineering and in environmental education generally (Morgan 1997): the degree to which consumers actually understand the energy and waste flows associated with their actions (and hence, for example, the correctness of their mental models) must affect how they respond to calls to change their behaviour. It is not, then, a major leap to consider inefficient or non-optimal operation of a consumer product by a user as an 'error' (whether mistake, slip or even simply laziness), and

NOTE: This is a preprint of an article whose final and definitive form will be published in the International Journal of Sustainable Engineering.

(C) 2008 Taylor \& Francis; International Journal of Sustainable Engineering is available online at: http://journalsonline.tandf.co.uk/ 
some constraint and affordance-based techniques as an appropriate basis for designing systems to alleviate the 'error'.

As a constraint, rationing of electricity, water, printer paper and so on might be considered, though not necessarily in a traditional sense - for example, making it a 'finite' resource from the user's point of view, within a limited context, by using the system to set limits or targets which can be exceeded, but only with extra work, costs, or commitment by the user. Coin-operated electricity meters fall into this category, but do not afford users the granular level of control over switching off individual devices that would be possible with a modern energy monitoring system. Lilley (2005) notes that Unilever's introduction of detergent tablets was in part a strategic tactic to attempt to ensure that users do not use more (or less) than the optimum amount of powder for each wash: pseudo-rationing in the form of portion control. Alternatively, resource sharing, as simulated by the 'Watt Watchers' system (Fischer et al, n.d.), places a constraint on the total amount of power (or other resource) being drawn at any moment in a system, causing users to co-operate with each other to moderate their consumption.

In terms of simple physical constraints, smaller sinks (or sinks which noticeably expand when they are filled beyond the "inscribed" capacity - such as the Cranfield University/Electrolux Smart Sink (Sherwin et al 1998) set an upper limit on the amount of water that can be used. Smaller rubbish bins (e.g. in a kitchen) make users more aware of the amount of waste they are generating, since the rubbish will have to be "taken out" more often, and hence may encourage sorting of waste for recycling and better compaction of waste.

Applying some of these affordance- and constraint-based techniques to the interaction between user and system, in the context of sustainable engineering and ecodesign, suggests a variety of specific implementations, some of which already exist, and some of which are purely speculative. It should be noted that a number of these implementations may, depending on how they are presented to users, become seen as excessive constraints on user behaviour, and as such, some persuasive methods (see section 2.2) may be more effective. For example, light fittings can be designed so that only approved low-energy components will fit, as is the case with the Eaton MEM BC3 range of lightbulbs and lamp-holders (Eaton Corp. 2003; Lockton 2007a), created to meet UK Building Regulations requirements for lighting points in new homes which will only accept low energy lamps (Office of the Deputy Prime Minister, 2002, p. 17) by compelling users to buy special 3-pin bayonet compact fluorescent bulbs (functionally identical to standard CFLs) and preventing the fitting of 2-pin bayonet bulbs entirely (whether incandescent filament or CFL). This creates an economic lock-in, not generally in the consumer's interest, and likely to provoke adverse reaction, as the readers' comments appended to Lockton (2007a) demonstrate.

\subsection{Persuasion and feedback}

While many affordance- and constraint-based techniques aim to 'force' user behaviour to conform to the designers' intent, persuading (or guiding) the user

NOTE: This is a preprint of an article whose final and definitive form will be published in the International Journal of Sustainable Engineering.

(C) 2008 Taylor \& Francis; International Journal of Sustainable Engineering is available online at: http://journalsonline.tandf.co.uk/ 
through less coercive methods also has a useful role in DwI thinking, and offers a somewhat 'friendlier' view of designing for behaviour change. 'Persuasive technology' as a discipline has been developed by Fogg (2003) and his team, primarily in the context of website and software design - captology ('computers as persuasive technology') being the term used - but there is significant potential for application in sustainable engineering and ecodesign.

Principles of persuasion which might be applied in a DwI context include the socalled 'six weapons of influence' outlined by Cialdini (2007) - reciprocation, commitment and consistency, social proof, authority, liking and scarcity. In general, these make use of cognitive biases and psychological heuristics to influence decisions and behaviour. Other methods of classifying persuasive strategies include the 'tools' approach used by Fogg (2003, p. 32-53) - reduction (simplification of a procedure), tunnelling (guidance through a procedure, such as a 'wizard'), tailoring (individual customisation), suggestion (intervention at the most opportune moment, or kairos), self-monitoring (allowing users to track their own behaviour), surveillance (allowing others to track users' behaviour) and conditioning (reinforcement). These are often employed in conjunction with one another.

Some persuasive principles have immediate applicability in encouraging users to reduce their energy or other resource use - for example, social proof could be applied by showing users of a system how their resource use compares with that of their peers or the group mean (normative messages). If users are made aware that they are using more energy or creating more waste than other 'similar' users, this may cause them to alter their behaviour. The issue is somewhat complicated by the boomerang effect - a form of rebound effect, whereby below-mean users may then increase their resource use to match what they perceive others are doing - but careful use of injunctive messages (stating approval or disapproval of the users' actions - which may also be used as part of a conditioning strategy) in addition to descriptive messages (e.g. a quantitative comparison of electricity use to the norm) has been shown to moderate this. For example, a study involving household electricity meters compared the effects that types of normative feedback had on subsequent electricity use; the addition of injunctive feedback to the descriptive resulted in a lack of the boomerang effect which occurred with descriptive feedback alone (Schultz et al 2007). Nevertheless, in many cases, extremely simple informational descriptive feedback - even more useful or easily visible markings or calibrations on a product - have the potential to make it easier for users to be more efficient. Electric kettles marked in 'cups' or 'mugs' in addition to millilitres and fluid ounces would seem to make it easier for users to fill up the correct amount of water for the task in hand.

Feedback is the key to many persuasive approaches: giving users an indication of how 'efficient' their behaviour is, whether by simple 'speedometer' or 'warning light' approaches (home energy monitors, or a suggested gear-change light as used on certain Volvo models (e.g. Volvo AB 1986)) or more involved persuasive techniques (e.g. energy monitors which allow users to review their recent usage, set targets to reduce this progressively, and suggest methods of doing this, or a driving style monitor such as the Foot-LITE project (The Engineer 2007) which offers similar

NOTE: This is a preprint of an article whose final and definitive form will be published in the International Journal of Sustainable Engineering.

(C) 2008 Taylor \& Francis; International Journal of Sustainable Engineering is available online at: http://journalsonline.tandf.co.uk/ 
abilities to review and set targets). Feedback can come from separate add-on monitoring devices, but may equally be built into the energy- or other resource-using products themselves in the form of displays and gauges: implementing kairos-type feedback at the point where users are actually able immediately to change their behaviour (rather than reviewing a log on a meter long after the event) offers some advantages. Some interesting examples of this thinking can be found in projects aiming to reduce users' water consumption by displaying persuasive feedback at the sink itself, notably the MIT Media Lab's WaterBot (Arroyo et al 2005), which employs a variety of persuasive techniques, and a number of projects by design students at Brunel University (Lockton 2007b).

Appropriate feedback can help users to develop more accurate mental models of how the engineered systems around them actually work, particularly in energy terms - for example, Sweden's Interactive Institute has done work including a computer game, 'The PowerHouse' which aims to teach teenagers more about energy-using behaviours in the home, and the impacts of using different appliances in different manners, through simulating a household, the appliances, and characters which interact with them (Bång et al 2006). The Institute's Static! Research project also led to a number of other interesting energy-use feedback concepts, including a power strip with an illuminated cord (where the intensity or pulse frequency of the illumination corresponds to the total current being drawn at the time) (Interactive Institute n.d.), an electric radiator using thirty-five $60 \mathrm{~W}$ incandescent lightbulbs (to illustrate clearly to users the significant heat by-product of incandescent filament household lighting) (Gyllensward et al 2006), and an 'Erratic Radio' which intentionally receives the $50 \mathrm{~Hz}$ signals from household electric appliances in the area, and uses these to affect the tuning of conventional radio stations, so that the sound quality deteriorates as more appliances are switched on in the room (Ernevi et al 2005).

The above examples help reveal the physical science behind everyday energy use; it is also appropriate to demonstrate to users the financial costs of their behaviour how much extra it will cost to switch a device on, how much it is costing per minute, how much it has cost in the past month, and so on - and this is something which the new generation of home energy monitors are well-placed to permit. For example, Ambient Devices' wirelessly networked 'Energy Joule' (Ambient Devices, n.d.) aims to persuade users to alter their 'discretionary' electricity use in response to signals about the current electricity cost per unit (e.g. reducing use at times of peak demand on the grid), in the process saving money.

\subsection{Context-based approaches}

Combining affordance- and constraint-based approaches with persuasive and feedback elements leads to context-based DwI techniques, where affordances, constraints or persuasive elements are selectively enabled or displayed depending on users' behaviour at the time. This is a subset of the field of intelligent machines, pervasive computing, smart objects, ambient informatics and so on: systems which

NOTE: This is a preprint of an article whose final and definitive form will be published in the International Journal of Sustainable Engineering.

(C) 2008 Taylor \& Francis; International Journal of Sustainable Engineering is available online at: http://journalsonline.tandf.co.uk/ 
automatically adapt their behaviour to information and circumstances in their environment, with artificial intelligence at the peak of the field.

From the sustainable engineering point of view, 'closed-loop feedback' systems which automatically correct user 'errors' (see section 2.1), where inefficient behaviour is defined as an error, are a step up from simple 'open-loop' feedback. This approach could involve continuous active monitoring of user behaviour, with 'correction' where necessary (analogous to electronic traction or stability control for cars), or systems which merely compensate for resource-intensive errors directly (e.g. a sink where the tap is switched off when the water reaches a certain level, rather than being allowed to run down the overflow).

If the error correction is sufficiently reliable, users may no longer need to perform certain interactions at all - a washing machine which switches to half-load settings automatically by weighing the load perhaps no longer needs a half-/full-load setting on the fascia. If it can read information about the clothes (e.g. from RFID tags) or even detect the amount of soiling, all the settings may be processed automatically, without user interaction. At the extreme of the context-based approach would be the 'optimum lifetime product', automatically disabling functions at the 'optimum' point in its life-cycle as part of a product lifetime optimisation strategy (Chalkley et al 2001) with a known amount of hours' use, a known amount of wear, and a known amount of energy used. This would ensure that products returned under manufacturers' take-back schemes are in predictable condition and replaced at the most efficient point to do so; this approach may be most appropriate for a product service system, where the user effectively rents the functions provided rather than owning the appliance outright.

\section{Further work}

It is hoped that this article provides a useful, if brief, introduction to a variety of approaches to designing for behaviour change in the context of a sustainable engineering. Based in part on some of the ideas outlined above, the authors are developing a 'suggestion toolkit', the Design with Intent Method, which will allow engineers, designers or other stakeholders to choose appropriate techniques applicable to different 'target behaviours'.

It is intended that the toolkit will be tested and refined through trials with designers, specifically working on sustainable engineering and ecodesign problems; selected solutions developed from these outcomes will then be prototyped and be compared in a series of user trials to determine quantitatively the actual resource-use reduction benefits resulting from different strategies.

NOTE: This is a preprint of an article whose final and definitive form will be published in the International Journal of Sustainable Engineering. 


\section{References}

Ambient Devices, n.d. Energy Joule [Online]. Available at http://www.ambientdevices.com/products/energyjoule.html [accessed 28 January 2008].

Arroyo, E., Bonanni, L., Selker, T., 2005. WaterBot: Exploring Feedback and Persuasive Techniques at the Sink. In Proceedings of the SIGCHI conference on Human Factors in Computing Systems, 2005, Portland, Oregon, p. 631 - 639.

Bång, M., Torstensson, C. and Katzeff, C., 2006. The PowerHouse: A Persuasive Computer Game Designed to Raise Awareness of Domestic Energy Consumption. In IJsselsteijn, W.A., de Kort, Y.A.W., Midden, C.J.H., Eggen, J.H. and van den Hoven, E.A.W.H. (eds.), Persuasive 2006 (LNCS 3962). Berlin: Springer Verlag, p. 123-132.

Chalkley, A.M., Harrison, D. and Billett, E., 2001. A review of product lifetime optimization as an environmental tool. In Culley, S., Duffy, A., McMahon, C. and Wallace, K., Design Methods for Performance and Sustainability (13th International Conference on Engineering Design, Glasgow, 2001). Chichester: John Wiley, p. 693-700.

Chase, R.B. and Stewart, D.M., 2007. Mistake-Proofing: Designing Errors Out, 2nd ed. John Grout/Lulu Press.

Cialdini, R.B., 2007. Influence: The Psychology of Persuasion. Rev. ed. New York: HarperBusiness.

Eaton Corporation, 2003. Revolutionary new lampholder for energy-efficient home lighting [Online]. Available at http://www.mem250.com/news/news01 jul03.htm [accessed 28 January 2008]

Ernevi, A., Palm, S. and Redström, J., 2005. Erratic Appliances and Energy Awareness. In Proceedings of Nordic Design Research Conference 'In the Making', May 2005, Copenhagen.

Fischer, J., Jones, S. and Kestner, J., n.d. Watt Watchers System Diagram [Online]. Available at http://www.jordanfischer.com/pdfs/watt_watchers.pdf [accessed 28 January 2008].

Fogg, B.J., 2003. Persuasive Technology: Using Computers to Change What We Think and Do. San Francisco: Morgan Kaufmann.

Gibson, J.J., 1979. The Ecological Approach to Visual Perception. Boston: Houghton Mifflin.

Gyllensward, M., Gustafsson, A. and Bång, M., 2006. Visualizing Energy Consumption of Radiators. In IJsselsteijn, W.A., de Kort, Y.A.W., Midden, C.J.H., Eggen, J.H. and van den Hoven, E.A.W.H. (eds.), Persuasive 2006 (LNCS 3962). Berlin: Springer Verlag, p. 167-170.

Interactive Institute, n.d. STATIC! Increasing Energy Awareness [Online]. Available at http://www.tii.se/static/index.htm [accessed 28 January 2008].

Jelsma, J., 2000. Design of Behaviour Steering Technology. In Proceedings of the International Summer Academy on Technology Studies 2000, Graz: IFZ, p. $121-132$

Jelsma, J., 2006. Designing 'Moralized' Products. In Verbeek, P.P., Slob, A. (eds.), User Behavior and Technology Development: Shaping Sustainable Relations Between Consumers and Technologies. Berlin: Springer, p. 221-231

NOTE: This is a preprint of an article whose final and definitive form will be published in the International Journal of Sustainable Engineering.

(C) 2008 Taylor \& Francis; International Journal of Sustainable Engineering is available online at: http://journalsonline.tandf.co.uk/ 
Kesan, J.P. \& Shah, R.C., 2006. Setting Software Defaults: Perspectives from Law, Computer Science and Behavioral Economics. Notre Dame Law Review, Vol. 82, p. $583-634$

Lilley, D., Lofthouse, V. and Bhamra, T., 2005. Towards Instinctive Sustainable Product Use (2nd International Conference, Sustainability: Creating the Culture, Aberdeen) [Online]. Available at http://hdl.handle.net/2134/1013 [accessed 28 January 2008].

Lockton, D., 2007a. A bright idea? [Online]. Available at http://architectures.danlockton.co.uk/2007/03/28/a-bright-idea/ [accessed 28 January 2008]

Lockton, D., 2007b. Changing behaviour: water meter taps [Online]. Available at http://architectures.danlockton.co.uk/2007/06/28/changing-behaviour-water-meter-taps/ [accessed 28 January 2008].

Lockton, D., 2008. What is Design with Intent? [Online]. Available at http://architectures.danlockton.co.uk/what-is-design-with-intent/ [accessed 28 Jan 2008]

Morgan, M.G., 1997. Public Perception, Understanding, and Values. In Richards, D.J., ed. The Industrial Green Game. Washington, DC: National Academy Press, p. 200-211.

Nikkan Kogyo Shimbun, Ltd. \& Factory Magazine, 1989. Poka-Yoke: Improving Product Quality by Preventing Defects. Portland: Productivity Press.

Norman, D.A., 1988. The Psychology of Everyday Things. New York: Basic Books.

Norman. D.A. and Lewis, C., 1986. Designing for error. In Norman, D.A. and Draper, S.W. (eds.), User-Centered System Design. Mahwah, NJ: Lawrence Erlbaum Associates.

Office of the Deputy Prime Minister, 2002. Approved Document L1, Conservation of Fuel and Power [Online]. Available at http://www.planningportal.gov.uk/uploads/br/BR_PDF_ADL1_2002.pdf [accessed 28 Jan 2008]

Product Creation Ltd., n.d. How to use Eco Kettle [Online]. Available at: http://www.ecokettle.com/AFTERSALES.htm [accessed 28 January 2008]

Schultz, P.W., Nolan, J.M., Cialdini, R.B., Goldstein, N.J. and Griskevicius, V., 2007. The Constructive, Destructive and Reconstructive Power of Social Norms. Psychological Science, 18 (5), p. 429-434.

Sherwin, C., Bhamra, T, and Evans, S., 1998. The 'eco-kitchen' project — using ecodesign to innovate. Journal of Sustainable Product Design, Issue 7.

Shingo, S., 1986. Zero Quality Control: Source Inspection and the Poka-Yoke System. Portland: Productivity Press.

Tefal UK Ltd., n.d. Tefal QuickCup [Online]. Available at: http://www.quickcup.co.uk [accessed 28 January 2008]

The Engineer, 2007. Foot off the pedal. The Engineer, 4 June [Online]. Available at http://www.theengineer.co.uk/Articles/Article.aspx?liArticleID=300099 [accessed 28 January 2008]

Volvo AB, 1986. Volvo 240 DL \& GL Owners' Handbook [Online]. Available at http://new.volvocars.com/ownersdocs/1986/1986_240/86240_02.htm [accessed 28 January 2008]

NOTE: This is a preprint of an article whose final and definitive form will be published in the International Journal of Sustainable Engineering.

(C) 2008 Taylor \& Francis; International Journal of Sustainable Engineering is available online at: http://journalsonline.tandf.co.uk/ 